Journal of Thermal Engineering, Vol. 7, No. 2, Special Issue 13 pp. 222-229, February, 2021

Yildiz Technical University Press, Istanbul, Turkey

\title{
A BAYESIAN NETWORK-BASED APPROACH FOR FAILURE ANALYSIS IN WEAPON INDUSTRY
}

\author{
Melih Yucesan ${ }^{1}$, Muhammet Gul2, ${ }^{2}$ Ali Fuat Guneri ${ }^{3}$
}

\begin{abstract}
Gun and rifle manufacturing contain various failures in the process of CNC machining, material supply, research \& development, infrastructure and, operator. Due to these failures, the enterprise is exposed to great economic losses and a decrease in competition in the global market. In addition, failures in production cause events that seriously threaten human health. Failure analysis can increase safety by determining the cause of potential errors and taking measures for identified errors in the life cycle of the products. Therefore, this study employs a Bayesian Network (BN)based modeling approach for capturing dependency among the basic events and obtaining top event probability. Firstly, a fault tree analysis (FTA) diagram is constructed, since its target is to pinpoint how basic event failures result in a top event (system) failure by an AND/OR logical gate. While, AND logical gate should take place in both cases, it is sufficient to realize one of the states in the OR logical gate. Then, a BN-based on fault tree transformation is applied. A case study in a leading weapon factory that produces various types of guns and rifles in the Black Sea region of Turkey is performed. For the application viewpoint, appropriate control measures can be taken into account to decrease the number of failed products based on the performed failure analysis.
\end{abstract}

\section{Keywords: Bayesian Network, Fault Tree Analysis, Weapon Industry}

\section{INTRODUCTION}

Failure analysis is very crucial in weapon industry facilities where the manufacture of components for defense systems and equipment is performed. Manufacturing of components such as gun and rifle contains various failures in the process of $\mathrm{CNC}$ machining, material supply, research \& development, infrastructure and operator. Failures in these processes evoke events that seriously threaten human health and facility safety. Failure analysis can increase safety by determining the cause of potential errors and taking measures for identified errors in the life cycle of the defense products [1,2]. In a weapon products manufacturing facility, failures such as non-conformity of tool coating, unsuitability of tool coating, tool size and tolerance error, supply of different products, error due to material production, missing operation, incorrect program, cooling fluid pressure, vibration and winding on the machine, power failure, storage ambient conditions, fragment of the part to the backing surface, reverse connection of the part, entering incomplete operations in the program, error in stacking parts, incorrect program operation, incorrect adjustment of the machine, and routine maintenance of the machine can occur due to mechanical, operator and ergonomic reasons. To this end, this study deals with a failure analysis study in weapon industry. A case study in a leading weapon factory that produces various types of guns and rifles in Black Sea region of Turkey is carried out.

Various methodologies have been proposed in the literature to assess riskiness and safety of processes of plants. Although classical quantitative risk assessment methods have great importance in process systems safety management, they involve a number of limitations especially emerged in dynamic risk/failure analysis [3]. These minuses are generally stemmed from their static nature and insufficiency of these methods in coping with uncertainty. Mostly applied classical risk assessment methods include bow-tie analysis, fault-tree analysis, event-tree analysis, matrix method, failure mode \& effect and critically analysis and so on. To overcome drawbacks of classical techniques,

This paper was recommended for publication in revised form by Regional Editor Hasan Köten

${ }^{1}$ Department of Mechanical Engineering, Munzur University, Tunceli, Turkey

${ }^{2}$ Department of Emergency Aid and Disaster Management, Munzur University, Tunceli, Turkey,

${ }^{3}$ Department of Industrial Engineering, Yıldız Technical University, Istanbul, Turkey,

E-mail address: melihyucesan@munzur.edu.tr, muhammetgul@munzur.edu.tr, guneri@yildiz.edu.tr

Orcid ids: 0000-0001-6148-4959, 0000-0002-5319-4289, 0000-0003-2525-7278

${ }^{*}$ Corresponding author

Manuscript Received 08 January 2020, Accepted 01 April 2020 
many scholars have merged classical methods with fuzzy theory or evidence theory, Petri nets and BN. One of the sophisticated techniques, BN, has several advantages as follows [4,5]: (1) They contain multi-state node variables capable of displaying multiple effect factors simultaneously, (2) They have an ability of dynamic probability updating, (3) They can easily demonstrate problems in which nodes represent random variables and arcs represent direct probabilistic dependencies among them, (4) They solve decision models with variables of several types.

BN has been applied to many different problems. Hosseini and Sarder [4] applied BN modelling to select an optimal charging station location for electric vehicles. This study also contributes to the literature by considering uncertainty in the choice of charging station location, qualitative and quantitative decision criteria and presenting BN as an effective decision support system in the field of energy. Liu and Liu [6] developed a new BN model and applied its reliability assessment of a subsea blowout preventer control system. Zarei et al. [3] improved BN by fuzzy sets. They applied fuzzy-based BN to an oil-gas station safety process. Expert opinion with fuzzy set theory was used in estimating probabilities of root events and consequences. Crisp and fuzzy-based BN models were then compared to reach a more realistic result and fuzzy-based BN was found superior against classical BN model. Similarly, Yazdi and Kabir [7] studied fuzzy-based BN for risk analysis of an ethylene transportation line. They used expert elicitation and fuzzy sets to overcome the uncertainty in failure data. They used BN modeling to capture the interdependence between events and provide a strong probabilistic rational under uncertainty conditions. Hamza and Hacene [8] discussed advantages of BN modelling against fault tree analysis in reliability and safety. They used a sample from the current literature to exemplify the application and compared results of both methods (FT and BN). Liu et al. [9] suggested a new approach to build BN from GO model. Tong et al. [5] investigated the mine gas explosion by employing BN. Based expert evaluations and mine gas accident data, they proposed a mine gas explosion model with twenty BN nodes. Smith et al. [10] examined and compared three safety analysis approaches named FT, BN, and the functional resonance analysis method (FRAM). To show the applicability of the three pillars-based model, a case study for a propane feed control system was performed. While Abimbola et al. [11] have applied BN to safety and risk analysis of managed pressure drilling operation, Yuan et al. [12] studied on risk analysis of dust explosion scenarios. Khakzad et al. [1] compared FT and BN as in Smith et al. [10]. Hanea and Ale [13] studied the risk of human fatality in building fires and developed a BN-based decision support system.

The present study applied BN as a failure analysis tool, which has not been studied thus far in the safety risk assessment literature. A real-world case-study was carried out in a leading weapon factory that produces various types of guns and rifles in Turkey, to determine significant failure factors, basic event probabilities, and top event probability for the process of scrapping. The previous literature is limited, and any similar attempt is not yet available that applied to failure assessment of the process industry using BN. In this study, we have also merged the mapping of FT model with BN so that the causes of an accident and conditional dependency can be easily analyzed in a holistic safety assessment.

Determining the cause of failure modes in technology-intensive production is very difficult for businesses. The weapon industry produces at low tolerance ranges and failure cannot be tolerated. Therefore, by using BN analysis, the factors causing the failure the most are determined. Thus, necessary measures can be taken to prevent high priority risk factors determined to reduce the number of scrap products, which is the main mistake.

\section{METHODOLOGY}

A BN (in another name belief network, Bayesian belief network or causal network) is an acyclic directed graph. It has nodes and directed arcs. Nodes represent random variables and arcs represent direct probabilistic dependencies among them [6]. There exist two types of nodes in a BN. The nodes with arrows directed into them are called child nodes. The nodes with arrows directed from them are called parent nodes [4]. In Figure 1, a small example to illustrate the basic characteristics of a BN.

In the example, Node "X" is a parent node. Nodes "Y" and " $Z$ " are child nodes. The prior probability "Y" or "Z" depends on the distribution probability of node " $\mathrm{X}$ ". A BN is mostly established via enough data. Since it is difficult to gather enough data, an expert knowledge is required. Data regarding prior probabilities for parent nodes 
are reachable by historical data. The probabilities of child nodes are from expert judgements. This is possible by such a method of Delphi method [5]. The equations regarding BN modelling are provided as follows:

$$
P\left(X_{1}, X_{2}, \ldots, X_{n}\right)=\prod_{1}^{n} P\left(X_{i} \mid \operatorname{parent}\left(X_{i}\right)\right) \quad(i=1 \text { to } n)
$$

Equation (1) refers to the joint probability of each node. It is calculated under conditional dependency.

$$
P(M \mid e)=\frac{P(e \mid M) P(M)}{P(e)}=\frac{P(e \mid M) P(M)}{\sum_{i=1}^{t} P\left(e \mid M_{i}\right) P\left(M_{i}\right)}
$$

The meaning of each notation used in Equation (2) are as follows:

- $e$ is a provided evidence

- $M$ is an event

- $P(M \mid e)$ is the posterior probability under $e$

- $P(M)$ is the prior probability of $M$

- $\quad P(e \mid M)$ is the evidence likelihood of $M$

- $\quad P(e)$ is the pre-defined posterior probability of $e$

- $\quad \sum_{i=1}^{t} P\left(e \mid M_{i}\right) P\left(M_{i}\right)$ is the joint probability distribution of $E$.

Equation (2) refers to the dynamic manner of a BN.

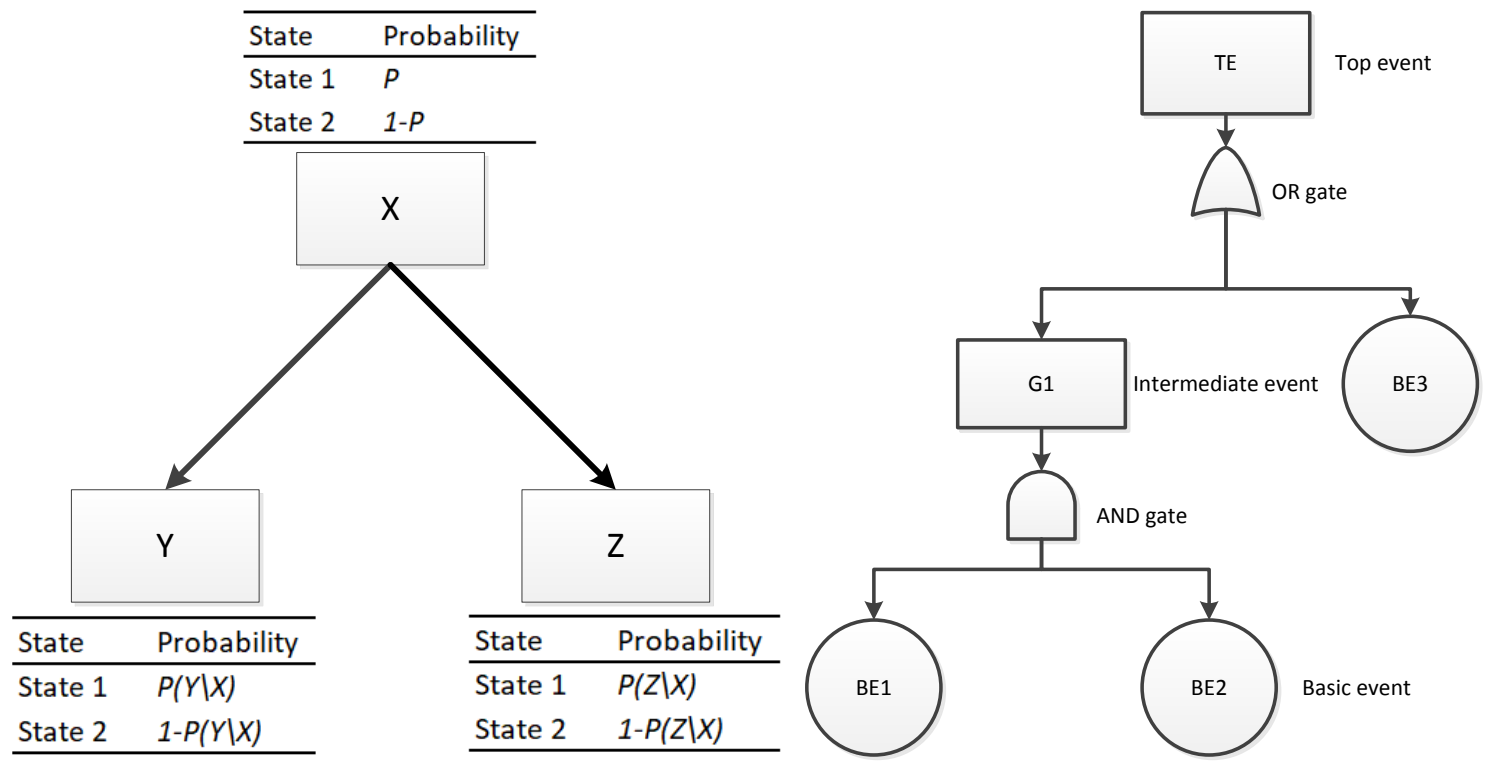

Figure 1. A basic representation of (a) BN, (b) FT

FTA helps determine the cause of failure or test the reliability of a system by stepping through a series of AND/OR logical operators [10]. A fault tree has two different node types: (1) event, (2) gate (Fig. 1). Events can be either basic events or intermediate events. Basic events occur independently, while intermediate events are caused by one or more events [14]. Apart from these two event types, an event type is introduced as the top event. It is called a cut-set and is the event being analyzed in modelling the failure of the system. Gates show how failures are spread in the system. In the current study, the structure of the system is mapped by the aid of FTA [15]. 


\section{BAYESIAN NETWORK DETERMINATION}

In this study, we recommend 31 nodes that have parent and child relationships with each other for representing scrap products for weapon factory from causes to consequences. All nodes and their connections were determined by evaluating expert opinions in the factory. Conditional probability tables (CPTs) are fundamentals of a BN and it can be obtained based on sufficient data. However, for many industries, there is no enough historical record or detailed statistical data [5]. In this study, we gather data from the factory by some experts. They provided us the CPTs in a twoweek time in November 2019 The conditional probability table of the nodes is based on the information in the enterprise database and the opinions of experts, as shown in Table 1. The states of Bayesian nodes and the prior probabilities of the independent nodes are given in Table 1. After CPTs of all nodes are determined, the initial BN of the factory is established in Fig. 2. BN probability calculation is calculated in Genle program due to its provide free academic version and user friendly interface. This program allows the creation of the Bayesian network, identifying dependent and independent events, fixing desired events, performing dynamic calculations and representing the relations between nodes with arrows. Each node can be represented by a graph or a diagram.

The states in the nodes are determined according to the characteristic of the node. For example, while the state of the power failure node is determined as yes or no, the suitability of the tool coating is determined as low, medium and high.

Table 1. State of the BN and initial probabilities

\begin{tabular}{|c|c|c|c|c|c|}
\hline Node & State & Probability & Node & State & Probability \\
\hline \multirow[t]{3}{*}{ Incompatibility of tool coating } & Low & 0.97 & \multirow[t]{3}{*}{ Storage environment conditions } & Low & 0.95 \\
\hline & Medium & 0.02 & & Medium & 0.03 \\
\hline & High & 0.01 & & High & 0.02 \\
\hline \multirow[t]{2}{*}{ Tool tolerance failure } & Yes & 0.05 & \multirow{2}{*}{$\begin{array}{l}\text { Non-connecting parts to the } \\
\text { surface gauge }\end{array}$} & Yes & 0.05 \\
\hline & No & 0.95 & & No & 0.95 \\
\hline \multirow[t]{2}{*}{ Supply of different products } & Yes & 0.01 & \multirow[t]{2}{*}{ Reverse connecting of the parts } & Yes & 0.03 \\
\hline & No & 0.99 & & No & 0.97 \\
\hline \multirow[t]{3}{*}{ Failure due to material production } & Low & 0.80 & \multirow{2}{*}{$\begin{array}{l}\text { Missing operation entry to the } \\
\text { program }\end{array}$} & Yes & 0.03 \\
\hline & Medium & 0.15 & & No & 0.97 \\
\hline & High & 0.05 & \multirow[t]{2}{*}{ Product stacking failure } & Yes & 0.005 \\
\hline \multirow[t]{2}{*}{ Missing operation } & Yes & 0.2 & & No & 0.995 \\
\hline & No & 0.8 & \multirow[t]{2}{*}{ Bonding of parts failure } & Yes & 0.01 \\
\hline \multirow[t]{2}{*}{ Incorrect program } & Yes & 0.05 & & No & 0.99 \\
\hline & No & 0.95 & \multirow[t]{2}{*}{ Improper tool selection } & Yes & 0.005 \\
\hline \multirow[t]{3}{*}{ Cooling fluid pressure } & Low & 0.98 & & No & 0.995 \\
\hline & Medium & 0.01 & \multirow{2}{*}{$\begin{array}{l}\text { Incorrect reference to the } \\
\text { machine tool }\end{array}$} & Yes & 0.07 \\
\hline & High & 0.01 & & No & 0.93 \\
\hline \multirow{2}{*}{$\begin{array}{l}\text { Failure caused by machine magazine } \\
\text { during tool movement }\end{array}$} & Yes & 0.05 & \multirow[t]{2}{*}{ Incorrect program execution } & Yes & 0.03 \\
\hline & No & 0.95 & & No & 0.97 \\
\hline \multirow[t]{3}{*}{ Vibration and winding in the machine } & Low & 0.97 & \multirow[t]{2}{*}{ Incorrect setting of the machine } & Yes & 0.001 \\
\hline & Medium & 0.02 & & No & 0.999 \\
\hline & High & 0.01 & \multirow{2}{*}{$\begin{array}{l}\text { Non-execution of routine } \\
\text { maintenance of the machine }\end{array}$} & Good & 0.95 \\
\hline \multirow[t]{2}{*}{ Power cut } & Yes & 0.005 & & $\mathrm{Bad}$ & 0.05 \\
\hline & No & 0.995 & & & \\
\hline
\end{tabular}

\section{RESULT AND DISCUSSION}

After constructing the BN in an FT structure, the probability of top event occurrence is obtained as 0.0941 . Bar chart of the probabilities is given in Figure 3. The states identified when creating the BN are represented by a separate color and can be named with the desired name. The generated BN allows the possibility of updating and event fixing to adapt to new situations. Probability update refers to the calculation of posterior probability as a result of event fixing. This is called scenario analysis. In the first scenario, it is estimated the changes if the evidence is Power cut=Yes. When this is the case, the final failure probability of the top event (the system) will become 0.1430 . That means the probability of the product being considered as scrap will increase to $14.30 \%$. The new obtained $\mathrm{BN}$ is demonstrated in 
Figure 4. According to the Figure 4, one can observe that this scenario effects a small part of the whole factory production process failures. It will be appreciated that not only one type of failure may be expected to occur simultaneously in a production process. Then it can be examined how a more comprehensive and complicated scenario will affect the final node.

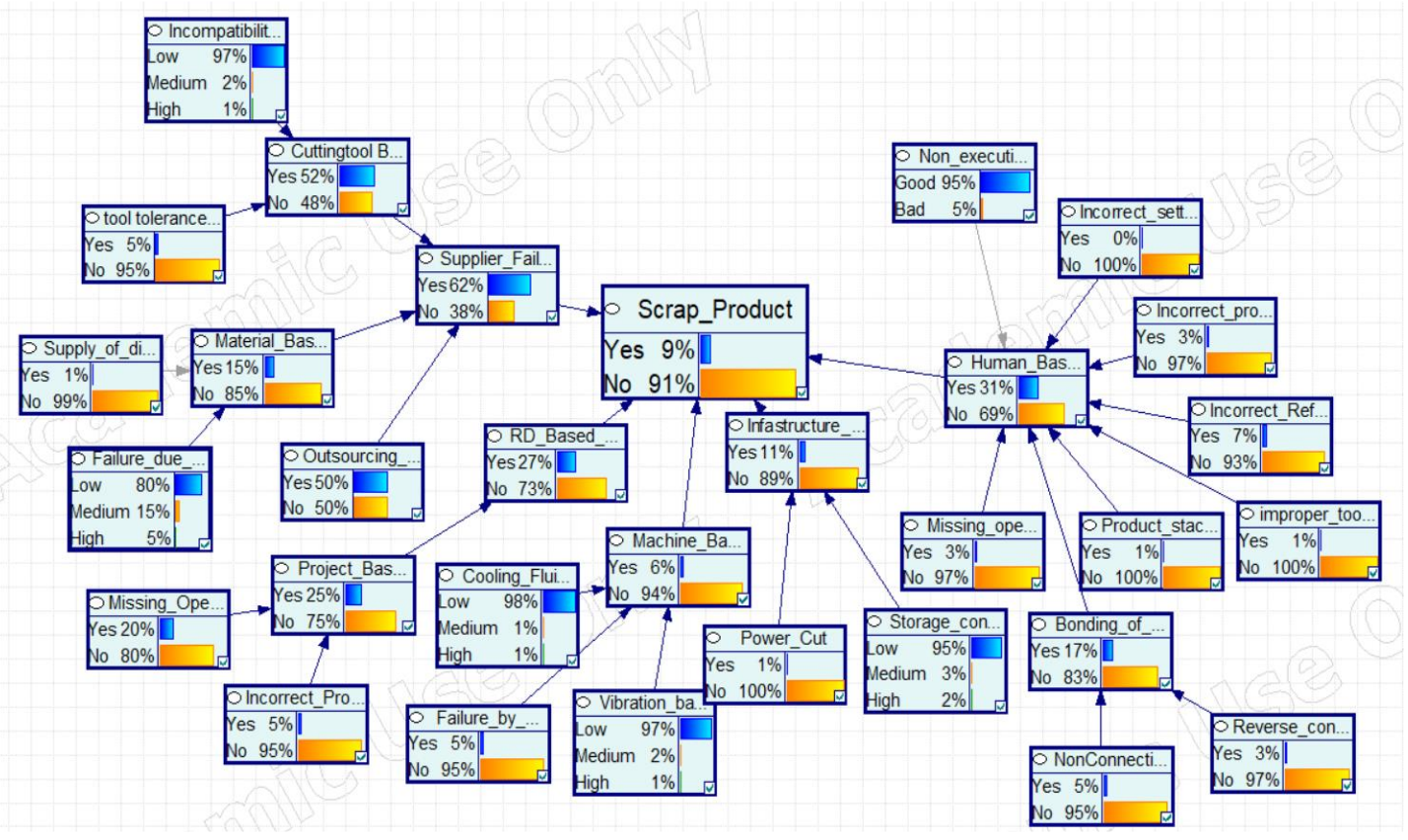

Figure 2. Initial BN of the factory (final node: scrap product)

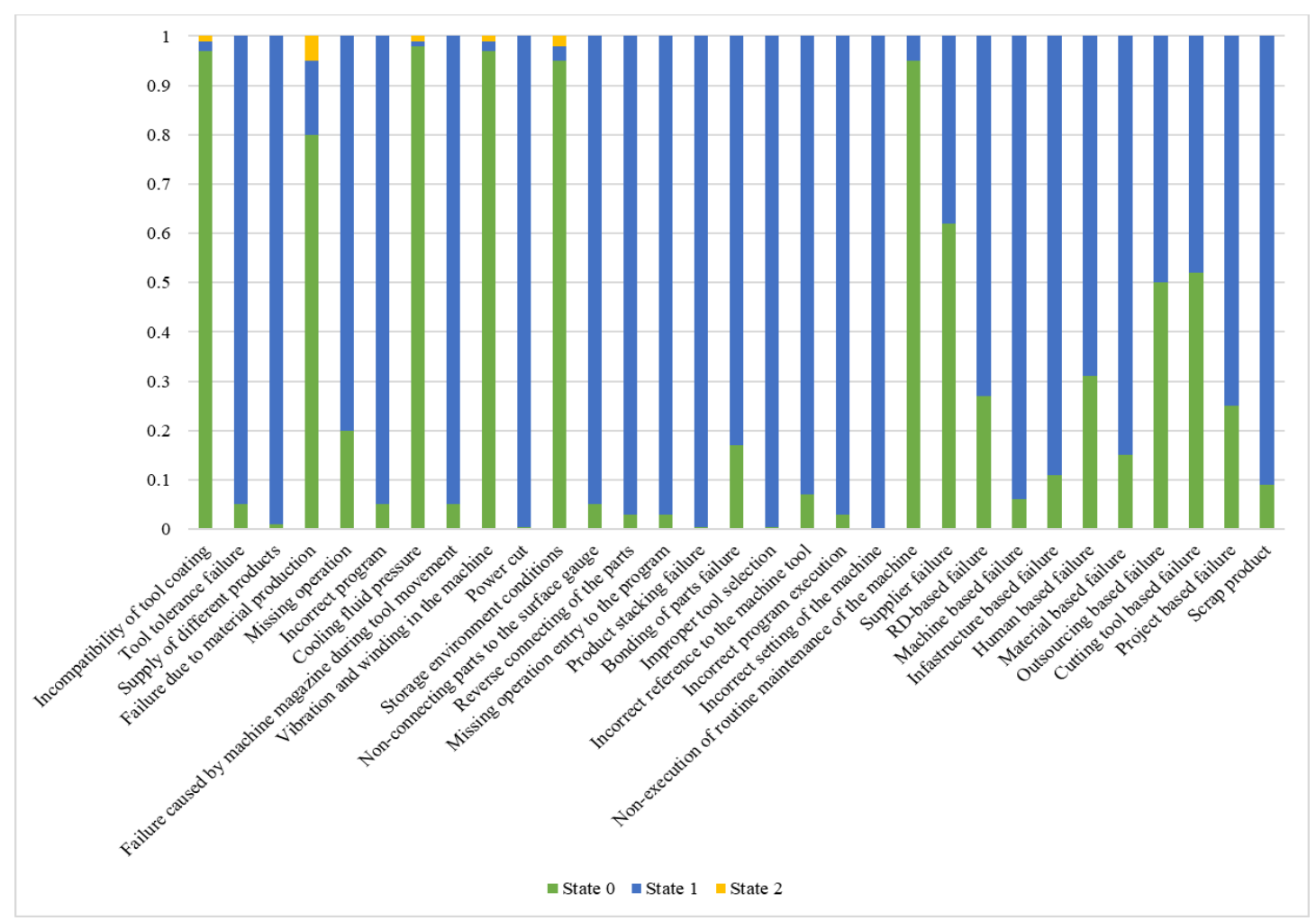

Figure 3. Posterior probabilities of the nodes in the way to the top event "scrap product 


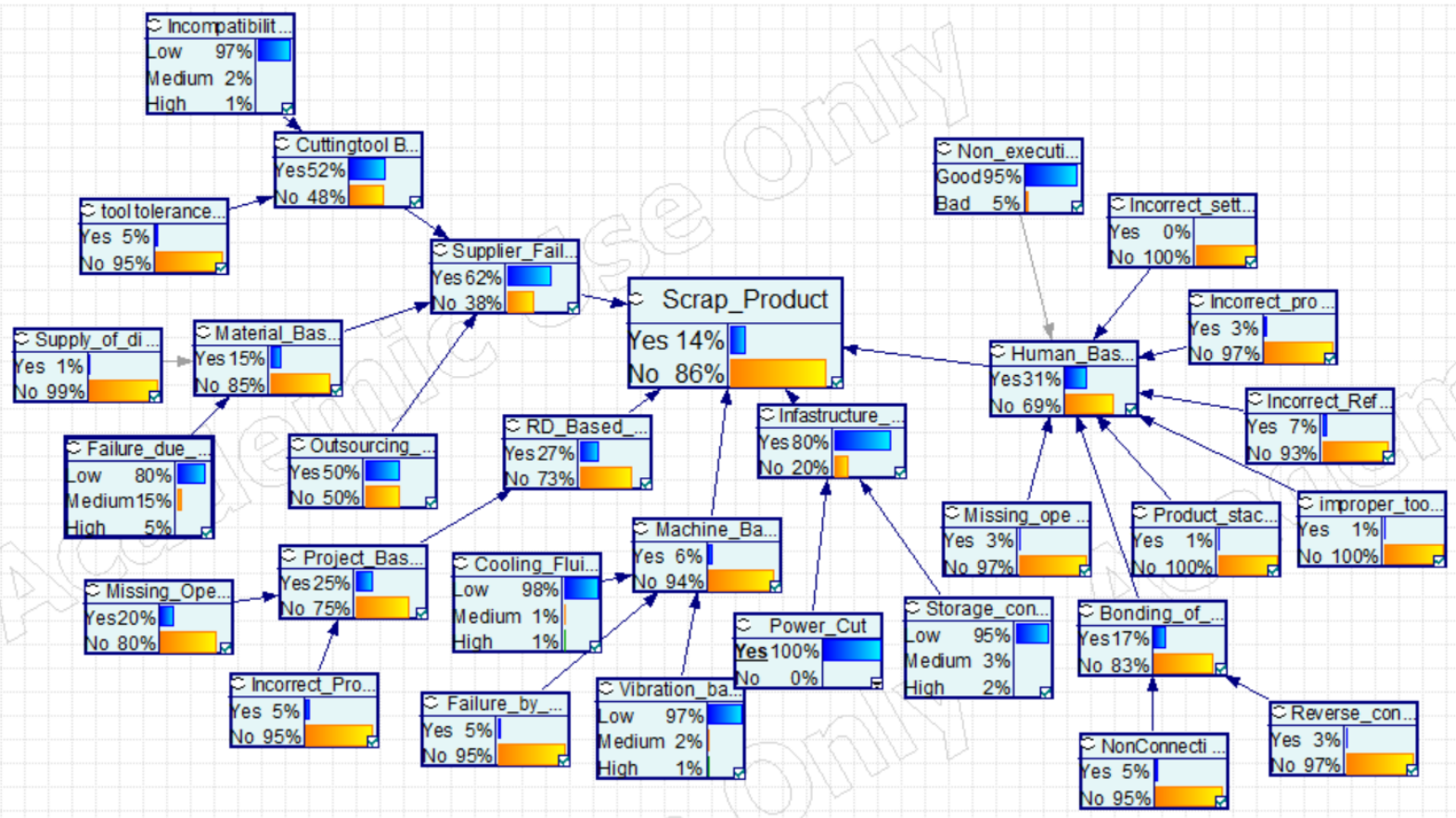

Figure 4. Posterior probability analysis in the first scenario "Power cut=Yes"

In the second scenario, attention has been paid to human and supplier related failures simultaneously associated with the scrap product in the production. Assuming that variables are assigned with the certain states in Figure 5, the effects of these mentioned factors on the top event are illustrated.

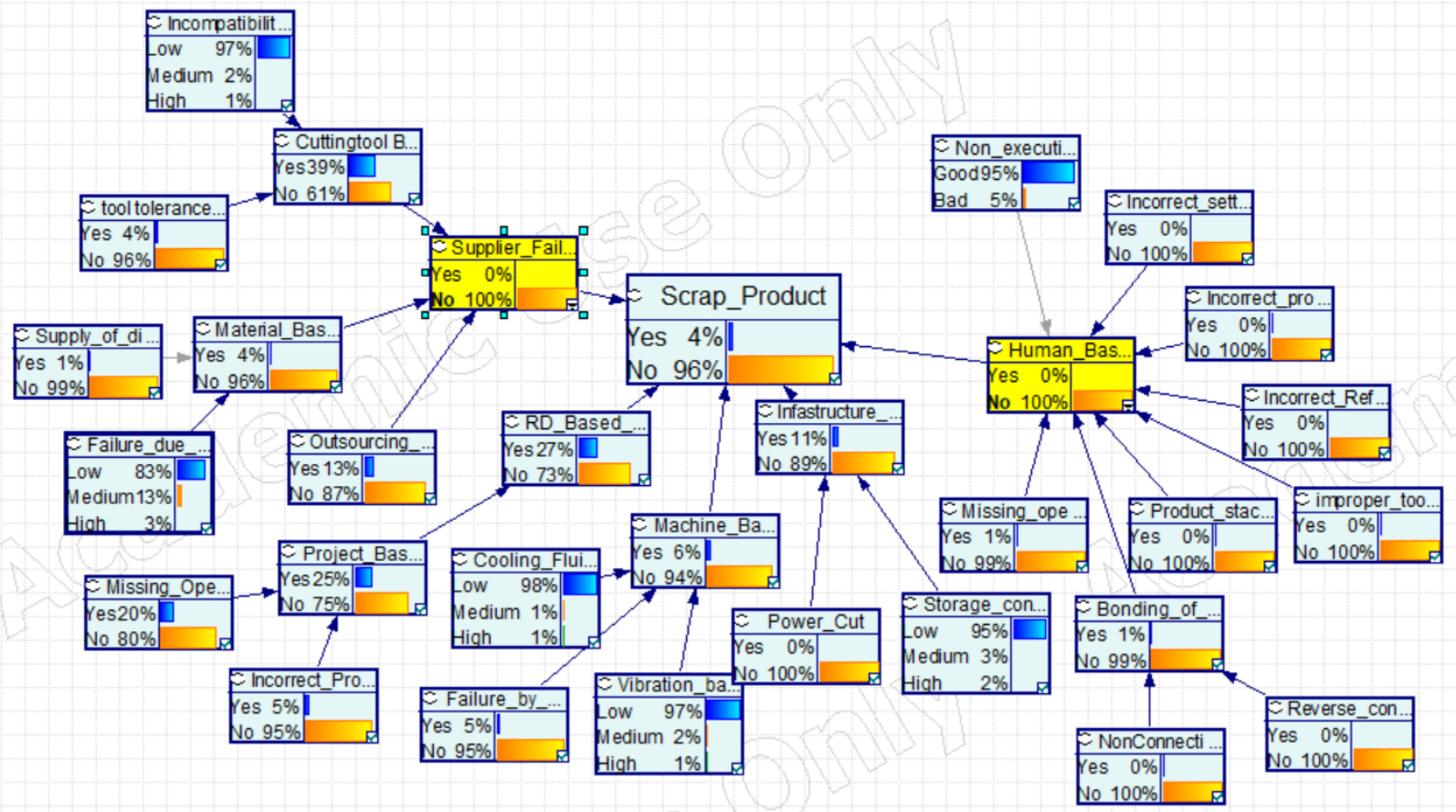

Figure 5. Posterior probability analysis in the second scenario 


\section{CONCLUSION}

The weapon industry is one of the leading industries in technology intensive, high precision and tight tolerance ranges. To make a sustainable production in this sector, it is necessary to produce quality and flawless production. In this respect, it is vital for businesses to identify the causes of failure modes and minimize them.

In this study, the probability of failure modes occurring in a weapon manufacturer was determined by using BN. BN analysis is used to determine the effect of each failure mode on the top event failure, which are called scrap product in this study. Thanks to the performed analysis, background information was provided in decision-making processes such as maintenance of machines, improvement of the production process and the use of new technologies in production. In addition, the performance of each machine or workstation was determined by this analysis. Through the existed study, a BN-based modeling approach for capturing dependency among the basic events and obtaining top event probability is proposed and applied to the failure analysis of a leading weapon factory that produces various types of guns and rifles in Turkey. From the application viewpoint, based on the failure and scenario analysis, appropriate safety measures can be taken to minimize the number of scrap products.

Via the constructed BN, cause and effect relationship between failure modes is revealed. This relationship can be easily updated and made it possible performing dynamic probability forecasts. Thus, the stakeholders of the observed factory have provided us the information regarding maintenance and machine replacement plans, allowing us to study different scenario analyzes. To this end two different scenario analyzes are designed. The effects of the handled factors on the top event are observed by simulating these scenarios.

The current study includes only the possibilities of occurrence of failure modes. Due to the fact that the company does not record the historical data on a regular basis, expert evaluations were made in determining the failure modes. In this context, in order to obtain a more effective failure mode analysis, it is considered to add the importance of failure modes to the model that will be proposed in future studies and evaluate the failure modes with linguistic expressions.

\section{REFERENCES}

[1] Khakzad N, Khan F, Amyotte, P. Safety analysis in process facilities: Comparison of fault tree and Bayesian network approaches. Reliab. Eng. Syst. Saf, 2011; 96:8: 925-32. https://doi.org/10.1016/j.ress.2011.03.012.

[2] Turhan C, Kazanasmaz T, Akkurt, GG. Performance indices of soft computing models to predict the heat load of buildings in terms of architectural indicators. J. Therm. Eng 2017; 3:4:1358-74. https://doi.org/10.18186/journal-of-thermal-engineering.330179.

[3] Zarei E, Khakzad N, Cozzani V, Reniers G. Safety analysis of process systems using Fuzzy Bayesian Network (FBN). J Loss Prev Process Ind 2019:57:7-16. https://doi.org/10.1016/j.jlp.2018.10.011.

[4] Hosseini S, Sarder MD. Development of a Bayesian network model for optimal site selection of electric vehicle charging station. Int J Elec Power 2019:105:110-22. https://doi.org/10.1016/j.ijepes.2018.08.011.

[5] Tong X, Fang W, Yuan S, Ma J, Bai Y. Application of Bayesian approach to the assessment of mine gas explosion. J Loss Prev Process Ind 2018:54:238-45. https://doi.org/10.1016/j.jlp.2018.04.003.

[6] Liu Z, Liu Y. A Bayesian network based method for reliability analysis of subsea blowout preventer control system. J Loss Prev Process Ind 2019:59:44-53. https://doi.org/10.1016/j.jlp.2019.03.004.

[7] Yazdi M, Kabir S. A fuzzy Bayesian network approach for risk analysis in process industries. Process Saf Environ 2017:111:507-19. https://doi.org/10.1016/j.psep.2017.08.015.

[8] Hamza Z, Hacene S. Reliability and safety analysis using fault tree and Bayesian networks. Integr ComputAid E 2019:11:1:73-86. https://doi.org/10.1504/IJCAET.2019.096720.

[9] Cai B, Liu Y, Liu Z, Chang Y, Jiang L. Risk analysis of subsea blowout preventer by mapping GO models into Bayesian networks. In Bayesian Networks for Reliability Engineering Singapore: Springer; 2020.

[10] Smith D, Veitch B, Khan F, Taylor R. Understanding industrial safety: Comparing Fault tree, Bayesian network, and FRAM approaches. J Loss Prev Process Ind 2017:45:88-101. https://doi.org/10.1016/j.jlp.2016.11.016.

[11] Abimbola M, Khan F, Khakzad N, Butt S. Safety and risk analysis of managed pressure drilling operation using Bayesian network. Saf. Sci 2015:76:133-44. https://doi.org/10.1016/j.ssci.2015.01.010.

[12] Yuan Z, Khakzad N, Khan F, Amyotte P. Risk analysis of dust explosion scenarios using Bayesian networks. Risk Anal. 2015:35:2:278-91. https://doi.org/10.1111/risa.12283. 
Journal of Thermal Engineering, Technical Note, Vol. 7, No. 2, Special Issue 13 pp. 222-229, February, 2021

[13] Hanea D, Ale B. Risk of human fatality in building fires: A decision tool using Bayesian networks. Fire Saf. J. 2009;44:5:704-10.

[14] Kang J, Sun L, Soares, CG. Fault Tree Analysis of floating offshore wind turbines. Renew. Energy 2019;133:1455-67. https://doi.org/10.1016/j.renene.2018.08.097.

[15] Badida P, Balasubramaniam, Y, Jayaprakash J. Risk evaluation of oil and natural gas pipelines due to natural hazards using fuzzy fault tree analysis. J Nat Gas Sci Eng 2019; 66:284-92. 